\title{
PLASMA OSCILLATIONS INSIDE SMALL FLUXTUBES
}

\author{
E. Wiehr * and G. Lustig \\ Institute for Astronomy, University of Graz/Austria \\ (* On leave from University Observatory, Göttingen/F.R.G.)
}

\begin{abstract}
Periodic Doppler motions inside small fluxtubes are determined from the zero-crossing point of the circular Zeeman polarization profile (Stokes-V=0). The gas fully participates on the known solar 5min oscillations without affecting amplitude or frequency. Averaging the oscillations, the gas is at rest in an absolute solar scale. Discrepancies with formerly observed redshifts are explained by the systematic westward displacement of the magnetic structure relative to the $\mathrm{Ca}^{+} \mathrm{K}$ bright point used for guiding.
\end{abstract}

\section{INTRODUCTION}

The investigation of oscillatory motions in presence of magnetic fields is of high interest for the understanding of the interaction between plasma and magnetic field. In the large scale sunspot magnetic fields, a significant damping of the amplitude of $5 \mathrm{~min}$ oscillations was reported by several authors (see e.g. Balthasar, Küveler, Wiehr; 1987). In contrast, small fluxtubes, known to be the origin of enhanced network and plage regions, seem to fully participate on the $5 \mathrm{~min}$ oscillations as observed by Wiehr (1985).

\section{OBSERVATIONS}

In order to verify the latter result at much higher spatial resolution, we took Zeeman spectra of a Ca+k bright point in the right and in the left handed circularly polarized light at the evacuated Gregory-Coude telescope on Tenerife/Spain. In 1986 both spectra were taken successively (Wiehr, 1986); in 1987 the use of Semel's (1980) polarimeter allowed strictly simultaneous exposures. The spatial resolution achleved during the total 
observing time of 15 and $18 \mathrm{~min}$, respectively, was always better than 1.5 arcsec. After photornetry at the Göttingen microdensitometer, corresponding right and left handid spectra were subtracted from each other, thus yielding the stokes- $V$ profile. The wavelength of the zero-crossing point, $V=0$, is given as a function of urre in Fig. 1.

\section{IRESULTS}

It can be seen that the 1986 as well as the 1987 data show well pronounced uscillatory Doppler shifts, the amplitudes being comparable to those known from the oscillations of non magnetic solar regions.

In addition, the $\mathrm{Fe} 6301.5$ line always shows smaller shifts than the $\mathrm{Fe}$ 6302.5 line. The wavelength difference of $150 \mathrm{~m} / \mathrm{s}$ equals the one found by Ballhatiar (1988) for the limb effect of both lines. Hence, when subtracting the corresponding granular blue shift for each line (introduced by the wavelength reference from the non-magnetic neighbourhood) the $v=0$ for both lint:s coincide. In addition, this correction procedure yields zero waveluigth displacement in an absolute scale for the points No.3 in 1986 and 1.6.6 in 1987 (both being almost free from oscillations). This indicates that, besides the oscillation, gas in the small fluxtube is at rest.

\section{CONCLUSION}

This result agrees with stenflo et al. (1987). Former results of downdrafts in small fluxtubes by wiehr (1985) are found to orginate from the iystematic westward tilt (Wiehr, 1978) of the magnetic maximum with respect to its corresponding Calk bright point used for punting. This displaces the fluxtube within the broad spectrograph slit used by wiehr (1985); for the particular time of observation during optimal seeing (9:30 through 10:30 local time) the westward direction corresponds to a redshift. As a consequence of the absence of downdrafts in small fluxtubes, the explanation of stokes- $V$ asymmetries in terms of a velocity gradient inside the fluxtube (as e.g. given by Stenflo et al., 1984) has to be reconsidered. A possible solution of ihis problem has been suggested by Grossmann-Doerth and Schuissler (1988) who consider the gas motion to be located outside line fluxtube; this would support the picture of a fluxtube imbedued in the downstreaming inter-granular lane. 


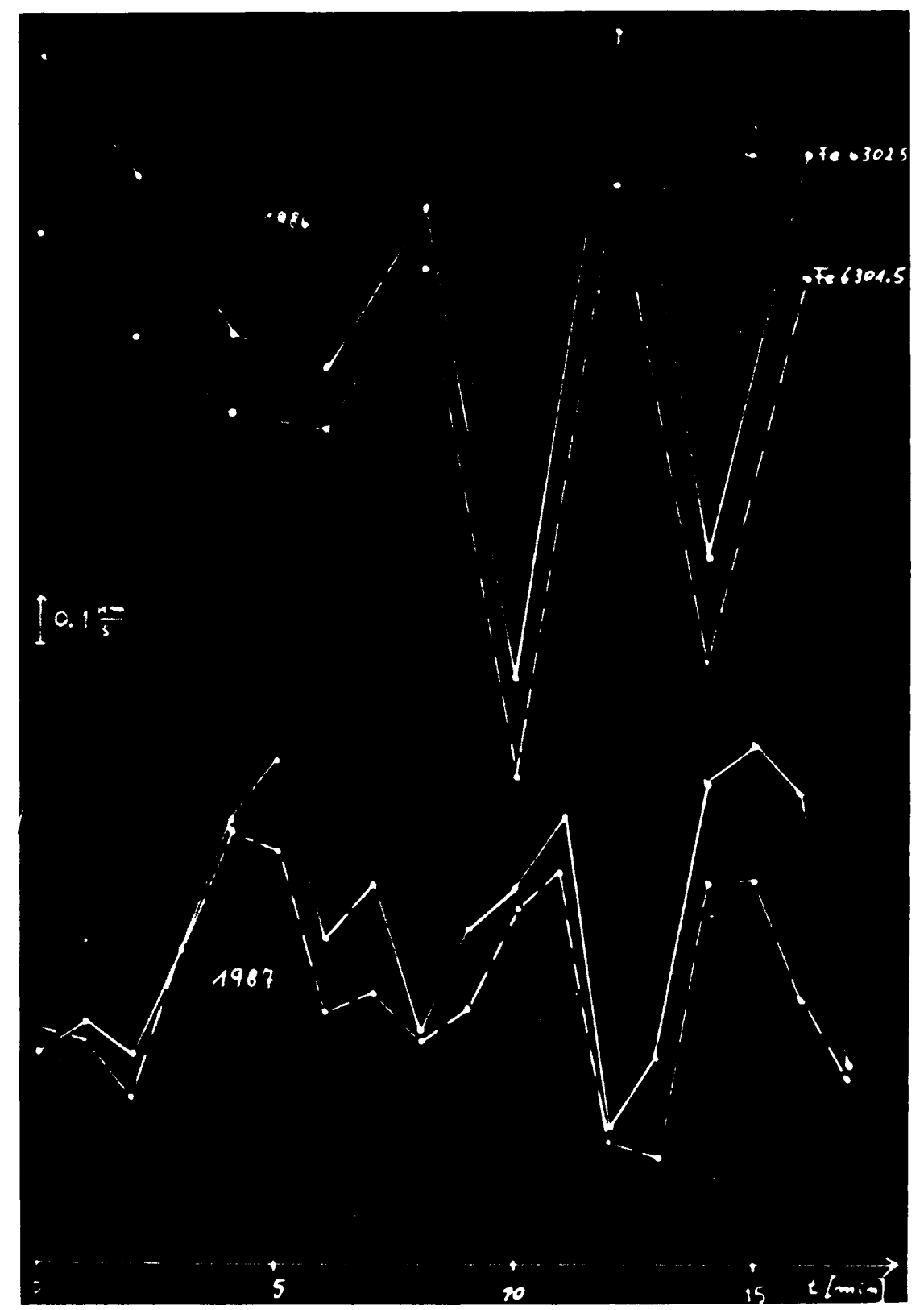

Fig. 1. Temporal variation of the inversion point $V=0$ of two Zeernan lines 
Balthasar, H., Küveler, G. and Wiehr, E.: 1987, Solar Phys. 112, 37

Balthasar, H.: 1988, Astron.Astrophys.Suppl.Ser. 72, 472

Grosmann-Doerth, U., Schüssler, M.: 1988, Astron.Astrophys. submitted

Sernel, M.: 1980, Astron.Astrophys. 91, 369

Stenflo, J.O., Harvey, J., Brault, J.W., Solanki, S.: 1984, Astron.Astrophys. 131, 333

Stenflo, J.O., Solanki, S., Harvey, J.: 1987, Astron.Astrophys. 171, 305

Wiehr, E.: 1978, Astron.Astrophys. 69, 279

Wiehr, E.: 1985, Astron.Astrophys. 149, 217

Wiehr, E.: 1986, the role of Fine Scale Magnetic Fields of the Solar Atmosphere, eds. E.H.Schröter, M.Vazquez, A.Wyller, Cambridge p.93 and p.354 